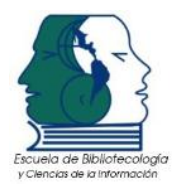

\title{
$>$

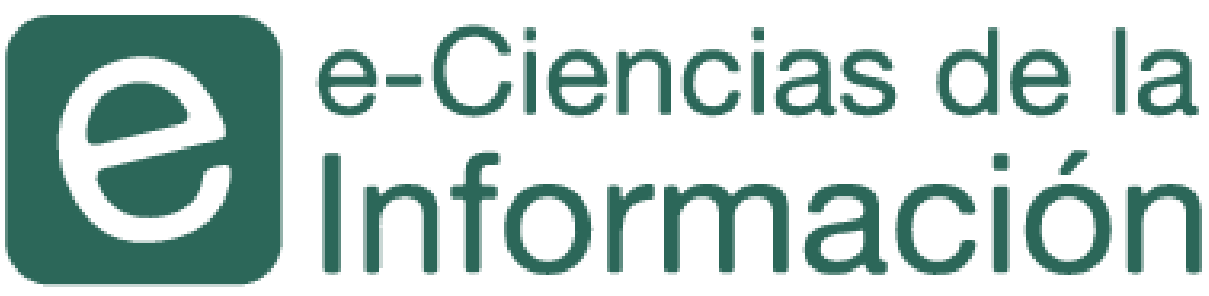

\section{Las bases de datos descriptivas: Un diseño de Modelo conceptual orientado a objetos}

$>$ Lizbeth Berenice Herrera Delgado

Publicado 01 de julio, 2017 / Artículo teórico

Revista electrónica semestral ISSN-1659-4142

\section{(C) (1) (2)}

Escuela de Bibliotecología y Ciencias de la Información Universidad de Costa Rica

Visite el sitio web de e-Ciencias de la Información 


\title{
$>$ Las bases de datos descriptivas: Un diseño de Modelo conceptual orientado a objetos
}

\author{
The descriptive database: A design of Conceptual object oriented model
}

Lizbeth Berenice Herrera Delgado'

\section{RESUMEN}

El presente artículo teórico es resultado de una investigación que conjuga la Ingeniería de Sistemas y la Bibliotecología; muestra una aproximación interdisciplinaria ante la problemática que presentan las bases de datos descriptivas de la biblioteca digital, problema generado por el cambio en los principios organizativos de la información y las demandas que el ambiente digital impone a las bibliotecas. El análisis conduce a un replanteamiento sobre la construcción de bases de datos descriptivas, migrando del Modelo entidad-relación (E-R) para adoptar el Modelo orientado a objetos (MOO) el cual en la actualidad es usado para la construcción y desarrollo programático de la web semántica. Por lo anterior, este artículo teórico propone un Modelo conceptual orientado a objetos bajo una metodología propia, la cual desarrolla el proceso para generar el modelo propuesto en dos etapas. La primera es de aproximación al problema, mientras que la segunda corresponde a la identificación de los elementos que incluye componentes de las normas Recursos, Descripción y Acceso (RDA) y observa los principios teóricos que establecen las FRBR (Requerimientos Funcionales para Registros Bibliográficos). Ya en su conjunto se analizan todos los datos para generar la propuesta de diseño de tres modelos conceptuales orientado a objetos para bases de datos descriptivas: 1) el Modelo de obra, 2) el Modelo de dominio y 3) el Modelo de instancia, para conformar una base de datos descriptiva más adecuada y propia de la biblioteca en esta era digital.

\section{ABSTRACT}

The present theoretical article is the result of an investigation that combines the Systems Engineering and the Library Sciences; it shows an interdisciplinary approach to the problem presented by the descriptive databases of the digital library, problematic generated by the change in the principles of organization of the information and the demands that the digital environment imposes to the libraries. The analysis leads to a rethinking about the construction of descriptive databases, migrating from the Entity-relationship Model (ER model) to adopt the Object-oriented Modeling (OOM), which is currently used for the construction and programmatic development of the semantic web. Therefore, this theoretical article proposes an Object-oriented Conceptual Modeling under its own methodology, which it develops the process to generate the proposed model in two stages. The first is to approach the problem, while the second corresponds to the identification of elements that includes component parts of the Resources, Description and Access (RDA) rules and observes the theoretical principles that establish the Functional Requirements for Bibliographic Records (FRBR). Already as a whole, all data are analyzed to generate the design proposal of three object-oriented conceptual modeling for descriptive databases: 1) the Work model, 2) the Domain model, and 3) the Instance model, to form a more adequate and descriptive database of the library in this digital era.

\section{Palabras clave}

Web semántica; Bibliotecas digitales; Mapas conceptuales; Bases de datos bibliográficas; Diseño de bases de datos.

\section{Keywords}

Semantic web; Digital libraries; Conceptual maps; Bibliographic databases; Databases design.

Recibido: 12 de diciembre, 2016

Aprobado: 05 de abril, 2017

1 Universidad Nacional Autónoma de México. Posgrado en Bibliotecología y Estudios de la Información. MÉXICO. Orcid: orcid.org/0000-0001-5605-1569. lizb2.herreradel@gmail.com 


\section{Introducción}

Para generar la propuesta de un modelo conceptual se requiere pasar por varias etapas de trabajo, mediante las cuales se definen jerarquías, se crean clases, se establecen relaciones y se identifican aquellos elementos que se incluirán en su diseño; todo ello sustentado en la investigación y la integración constante de nuevos datos para la identificación de las tareas generales y sustantivas a representarse y relacionarse, logrando la conformación de un todo llamado sistema.

Aun cuando varios autores hablan sobre la elaboración de modelos en los ámbitos de la ingeniería de sistemas, los hallazgos indican que no existe una metodología única o general. En su lugar, se han presentado metodologías particulares sugeridas por autores como Bertalanffy (1976), Batini, Ceri y Navathe (1992), Booch (2001), Casas Roma y Conesa I Caralt (2013) y Ashby (1957) -solo por mencionar algunos-, cuya hechura se centra en casos específicos para el diseño de modelos conceptuales, funcionales y/o con fines de aplicación práctica dentro del ámbito informático.

Ante esta situación, y para este artículo, se toma como base la metodología propuesta por Ashby para la creación de un modelo conceptual adicionándole el enfoque y los principios que establece la Teoría general de sistemas de Ludwing von Bertalanffy ${ }^{2}$; con ello se logra establecer una metodología propia mediante la cual se generó el diseño del modelo aquí presentado, transitando por dos etapas:

En una primera etapa se desarrolla una aproximación del problema, analizando las condiciones generales del contexto o entorno: conocimiento del fenómeno a modelar, conocimiento de programas -internacionales, nacionales, regionales- afines preexistentes, conciencia pública y capacidad de integración. Es por ello que se abordarán las tecnologías de la información y la comunicación y el análisis de las condiciones del "sitio" (para identificar su estado, establecer un inventario de sus necesidades y prioridades para después definir el área de estudio y la necesidad de datos) haciendo referencia directa a la web, sus capas y el tratamiento de la información. También se abordarán las reglas de lenguaje para la construcción de la web semántica para finalmente definir la filosofía de modelación; es decir seleccionar el modelo que se aplicará en la simulación de una realidad específica, a través tanto de los lineamientos y códigos de catalogación, así como de los formatos de codificación en conjunto con las bases de datos bibliográficas y su diseño estructural.

En una segunda etapa se identifican los elementos que conformarán al modelo, es decir: las clases, los objetos, sus relaciones e instancias. Para esto se considerará la organización señalada en las FRBR (obra, expresión, manifestación e ítem) a través del desarrollo de las RDA (Taylor, 2000). Además, se determina la DTD de las instancias (respetando la sintaxis especificada por el lenguaje XML), se establece el orden o las jerarquías de generalización

\footnotetext{
2 En términos generales un sistema se comprende como el conjunto de elementos interrelacionados. El cual puede ser concreto, a razón de su conformación, elementos y relaciones establecidas entre sí; o puede ser abstracto, gracias a la naturaleza de sus elementos como: conceptos abstractos y reglas lógicas que les relacionen. Ambos tipos de sistemas, suelen representarse mediante un modelo de funcionamiento o estructural; que a la larga bien puede constituir un software de computadora.
} 
(mediante las clases, sus nombres y relaciones), se bosqueja el modelo gráfico (la presentación del modelo conceptual como producto final) y se desarrolla el esquema conceptual de objetos y sus relaciones (como esbozo esquematizado a partir de diferentes principios de orden y funcionamiento). El resultado de esto es la presentación de tres modelos: 1) el Modelo de obra, 2) el Modelo de dominio y 3) el Modelo de instancia.

\section{Primera etapa: las tecnologías de la información y la comunicación}

En su artículo titulado Analysis of the Interdisciplinary Nature of Information and Library Science, Gila Prebor (2010) a lo largo de su trabajo, establece una división temática en los estudios de la información, al tiempo que afirma que:

Library and Information Science (LIS) is a field that has undergone many changes in its relatively short history. Highly interdisciplinary nature, LIS is affected by the incesante evolution of technologies [La biblioteca y la ciencia de la información es un campo que se ha sometido a muchos cambios en su relativa corta vida. Altamente interdisciplinaria por naturaleza, LIS es afectada por la incesante evolución de las tecnologías]. (Prebor, 2010, p. 256)

En efecto, las Tecnologías de la información y la comunicación (TIC) se han convertido rápidamente en instrumentos ubicuos de nuestra vida cotidiana. Ello responde al enorme potencial que suponen a través de sus innovaciones, pues los desarrollos que hasta ahora hemos podido conocer, van más allá de las mejoras que incrementan los beneficios de los productos y/o de los servicios existentes. Es así como, gracias a la evolución de las TIC, se están creando herramientas y servicios de los cuales -aparentemente- depende nuestro crecimiento inteligente.

Dada su integración en casi todos los ámbitos es que se puede observar la generación de drásticas transformaciones de los servicios que se convierten en requerimientos en los modelos de negocios que remplazan las formas tradicionales de las tareas y los procedimientos inmersos en un ámbito totalmente digital; esto mismo es lo que se pretende llevar al ámbito bibliotecológico.

En el caso de la biblioteca (cuya existencia se remonta a la época clásica de la cultura humana y cuya permanencia continúa hasta nuestros días) se ha sumado la inclusión y uso de las TIC, y por ende le ha permitido reafirmarse como una importante institución que apoyan la toma de decisión informada en todos los ámbitos. Claro ejemplo corresponde a la educación a distancia, tanto formal ${ }^{3}$, como informal (caso específico de los long learners, como uno de los tipos de usuarios, identificados como demandantes de estos servicios), y que decir de la investigación con fines diversos que responden a uno o más ámbitos como el social, cultural, económico o

3 Ofrecido por instituciones de educación que, aprovechando las diversas plataformas y sus herramientas creadas en favor de los cursos, diplomados o programas institucionales educativos, cuyo ejemplo más popular es el MOOC (Massive Online Open Courses) o COMA (Curso Online Masivo Abierto). 
académico, donde la biblioteca siempre se hace presente ya sea como apoyo de las actividades académicas, o como ofertante directa de recursos de información (ya que selecciona, organiza, describe, localiza y brinda acceso a los diferentes recursos de información además de resguardar el patrimonio documental de todas las culturas existentes y cuyo objetivo es el de volverla asequible al usuario).

Es por ello que resulta clara su importancia y su trascendencia respecto al desarrollo potencial a través de la adopción de las tecnologías emergentes que apoyen los procesos, tareas y servicios, así como sus portales web; por lo que las TIC son concebidas como aquellas herramientas que permiten eficientar aquellas tareas concatenadas e interdepartamentales desarrolladas por el bibliotecólogo.

\subsection{La web, sus capas y el tratamiento de la información}

En la biblioteca se concibe a la web por un lado como un ambiente alterno (paralelo al físico) y simultáneo, y también como una plataforma de y para los programas de computadora, ya que los aloja, los estructura y dota de funcionalidad. Teniendo presente lo anterior, y de acuerdo con la información presentada en el sitio web World Wide Web Size de Maurice de Kunder (2016), dónde se afirma que al 27 de mayo de 2017 se cuenta con por lo menos 4.49 billones de páginas indizadas en la web (Kunder, 2016), por lo que se calcula que su tamaño traducido al peso total en terabytes, sobrepasa los 5 millones ${ }^{4}$ de los cuales un $99.7 \%$ conformará la web profunda por no ser visible y solo el $0.3 \%$ hará visible la información de interés general, páginas oficiales de instituciones, libros, trabajos académicos, música, juegos, diversos tipos de entretenimiento y las redes sociales.

La exploración a través de ambas capas de la web se realiza gracias a los navegadores como Internet Explorer, Mozilla Firefox, Google Chrome, Opera, Safari, mientras que la visibilidad de la información se debe a la indexación de páginas y su explotación a través de los motores de búsqueda como son Google, Yahoo, Bing, YouTube, Twitter, Altavista, etc.

En este sentido las bibliotecas y su información, se localizan dentro de la web profunda en función de la configuración que tienen y debido a que su puerta de entrada son los propios motores de búsqueda del OPAC lo que se traduce en un cierre parcial del acceso a la información. Esta situación se ha intentado aminorar mediante el surgimiento de buscadores especializados como Google Académico, metabuscadores y sistemas de descubrimiento, así como con el uso de estándares internacionales y protocolos de intercambio de información tales como: OAI-PMH, XML, etc., pero su usabilidad todavía es muy reducida en comparación al uso existente de los buscadores comerciales; esta idea es consistente con autores como Játiva Miralles (2009) quien además cita a Antelman, Lynema, Pace (2006).

Ante un panorama como este resulta necesario comprender la construcción estructural de las bases de datos utilizadas por los sistemas integrales de información, ya que son la clave esencial pues contienen la información bibliográfica descriptiva con la que se trabaja, se 
brindan servicios y se da acceso a los recursos informativos al usuario a través de sus propios motores de búsqueda.

Por su parte, la web semántica surge con la visión de lograr la disposición de datos en ella contenidos, definidos y vinculados de tal forma que puedan ser descubiertos por las máquinas, y no solamente con el objetivo de ser visualizados sino con la posibilidad de automatizar tareas, integrar datos y reutilizarlos con cualquier aplicación actual o emergente, creando servicios cada vez más inteligentes y adaptativos.

Dentro del plan estratégico del desarrollo de la web semántica se cuenta con algunos resultados elementales de mediana efectividad tales como la creación de aplicaciones de software inteligente llamados agente ${ }^{5}$, capaces de procesar y operar los recursos electrónicos de información a nivel semántico (Berners-Lee, Hendler y Lassila, 2001).

De acuerdo a Alegsa (2016, párr. 2): "El concepto de un agente provee una forma conveniente y poderosa de describir una compleja entidad de software, que es capaz de actuar con cierto grado de autonomía, para cumplir tareas en representación de personas". En este sentido un agente es definido por su propio comportamiento cuyas características esenciales enunciadas por Alegsa son:

- Persistencia: el código se ejecuta continuamente y decide por sí mismo cuándo debería llevar acabo alguna actividad.

- Autonomía: capacidad de seleccionar tareas, priorizarlas, tomar decisiones sin intervención humana, etc.

- Capacidad o habilidad social: tomar otros componentes, a través de coordinación, comunicación y colaboración, que puedan colaborar en una tarea.

- Reactividad: perciben el contexto en el cual operan y reaccionan a éste apropiadamente. (2016, párr. 4)

Mientras que las denominaciones respecto a su funcionalidad según Alegsa son:

-Agentes inteligentes (agentes que utilizan inteligencia artificial, como aprendizaje y razonamiento).

-Agentes autónomos (agentes capaces de modificar la forma en que ellos llevan a cabo sus objetivos).

-Agentes distribuidos (agentes que son ejecutados en distintas máquinas). -Sistemas multiagentes (agentes distribuidos que no tienen la capacidad de alcanzar sus objetivos solos y por esto deben comunicarse).

-Agentes móviles (agentes que pueden trasladar su ejecución a diferentes procesadores). (2016, párr. 5)

5 Entidades o abstracciones de programas que cumplen el papel de mediadores de información; gracias a cierta autoridad y, de acuerdo con el Diccionario de informática, internet, tecnologías y computación on line, sirven "para decidir cuándo una acción es apropiada" y si lo es; se activándose en el momento. En otras palabras, un agente es una idea, un concepto, similar a los métodos, funciones y objetivos en la Programación orientada a objetos (Alegsa, 2016) 
Estos agentes son concebidos como entidades de software cuyas capacidades se abocan a los procesos de recolección, filtrado, procesamiento de información e inferencias de manera tanto semiautónomas como automáticas.

Para hablar de una web semántica se requiere desarrollar la modificación de los datos -es decir volverlos semánticos- mediante la transferencia recíproca de información, a través de líneas de intercambio de documentos electrónicos. El número de las formas del documento que se ofrecen son concebidas como equivalentes digitales de los documentos en papel. En este caso, la semántica de cada forma se define por las especificaciones del documento legible por el ser humano.

Es así que la web centra su mirada en la semántica y sus principios, para conducir el diseño y la aplicación de descriptores de lenguaje, ontologías ${ }^{6}$, reglas de lenguaje, razonadores y lógica basada en lenguajes con los que se describe y registra a los recursos públicos en la red. Su finalidad es dotarlos de significado al ser registrados como parte de los metadatos ${ }^{7}$ y de esta forma permitir la recuperación de datos, de tal forma que sean eficientes los procesos inteligentes de información y se genere la entrega oportuna de esta a los usuarios demandantes.

Estos puntos de relación deberán ser el primer paso para la estructura y creación de ontologías con un alto contenido semántico, y justamente éstas son el eslabón principal de la web semántica pues facilitarán las relaciones que la información posea, potencializando su recuperación a través de la web.

\section{Segunda etapa: de los lineamientos y códigos de catalogación}

En la historia de la Bibliotecología, se ha pasado por el desarrollo de diferentes normas que describen y organizan la información como son las ISBD o las AACR2, el formato MARC y sus múltiples versiones, las directrices en la ordenación de autoridades y referencias, los estándares de comunicación como el protocolo TCP/IP o el Z39.50 para facilitar el intercambio de información entre aplicaciones de sistemas, los números internacionales normalizados como el ISBN, ISSN, DOI, etc., para identificar inequívocamente los documentos. Estos han intervenido, de una u otra forma, en el desarrollo de los sistemas, la forma de organizar e identificar la información y las directrices con que se construyen las bases de datos.

Hemos observado desarrollos en los lineamientos y códigos de catalogación, en los que se considera el aspecto que establece un orden en el mundo del saber, de las ideas, de la información y de todas las cosas que en él existen. El ser humano se ha dedicado a desarrollar formas de estructuración basándose en múltiples y distintos criterios, siempre tratando de crear

6 Parafraseando a Sánchez López (2007), es factible afirmar que la ontología es la teoría de objetos en términos de criterios, que nos permiten distribuir entre diferentes tipos de objetos y sus relaciones, dependencias y propiedades.

7 De acuerdo a Berners-Lee, los metadatos son información inteligible para el ordenador sobre los recursos web u otras cosas (TED, 2009); de acuerdo a lo anterior, los metadatos se encuentran presentes tanto en los contenidos descriptivos propios de los datos bibliográficos, así como en las estructuras de programación de los sistemas como tags identificadores. 
una fórmula infalible, eficiente y universal; es por esto que se comprende que la organización y sus lineamientos resulten uno de los temas de mayor interés para nuestra profesión.

En este sentido, la propuesta más actual, mejor recibida y aceptada en el ámbito bibliotecológico corresponde a las Resource, Description and Access (Recursos, descripción y acceso, como su traducción oficial al español) $\operatorname{RDA}^{8}$ cuya base se establece a través del modelo conceptual Functional Requirements For Bibliographic Records (Requerimientos Funcionales de los Registros Bibliográficos, como su traducción oficial al español; por sus siglas en inglés: FRBR) ${ }^{9}$, el cual es un nuevo modelo conceptual de organización de los datos pertenecientes a los recursos de información (cuya pretensión es minimizar las diferencias actuales en pro de una mejor y más eficaz recuperación) centrados en la variedad que exhiben los recursos digitales.

Las FRBR refuerzan los objetivos básicos de la catalogación y la importancia de las relaciones, esto ayuda a los usuarios, ya que mejora la búsqueda, la localización, a identificar y seleccionar la información deseada (Tillet, 2004). Por su parte, las RDA presentan una estructura en la que se reúnen en principio estas tareas básicas del usuario, con el modelo conceptual de las bases de datos y con ello se establece la operación del universo bibliográfico a través de dos vehículos: la identificación de elementos y el establecimiento de sus vínculos relacionales. Ambos vehículos son tomados tal como se han expuesto, y se trasladarán en la interpretación de los elementos propios del modelo aquí desarrollado.

Esto permitirá reunir aquellos elementos que comparten, por ejemplo, la misma intelectualidad y/o un contenido artístico, ofreciendo una nueva visión de orden y concepción del universo bibliográfico. Razón por la cual existe un conjunto de datos o atributos que resultan obligatorios para el nivel de registro bibliográfico nacional, concebidos como los elementos nucleares para el acceso, descripción bibliográfica en los OPAC de las bibliotecas, sus sistemas y la observación de las RDA.

El Joint Steering Committee for Development of RDA (JSC) a través de las RDA, pretende sentar tres precedentes:

[1] Provide a consistent, flexible and extrensible framework for both the technical and content description of all types of resources and all types of content ... [2] Enable users to find, identify, select, and obtain resources appropriate to their information needs ... [3] Optimised for use as an online tool (although a print edition will also be published) [Proveer consistencia, flexibilidad y establecer un marco general tanto para aspectos técnicos, como para la descripción de contenidos de todo tipo de recursos y contenidos ... Establecer descripciones y puntos de acceso que

8 Este es el nuevo código de descripción y acceso a recursos de información que busca sustituir las AACR2, actualmente en uso, a modo de prueba en bibliotecas como la de la Library of Congress, que promete elevar la eficiencia en la catalogación sin discriminar el tipo de soporte propio del recurso.

9 Parte los FRBR establecen los atributos de las obras (entidades) como características que contribuyen a la representación de una obra, con los cuales se identifican los elementos inherentes a ella (centímetros, título de la cubierta, chaleco, etc.) y también se incluyen los insumos externos (número del catálogo temático en una obra musical o datos de referencia provenientes de una fuente externa). 
permitan a los usuarios encontrar, identificar, seleccionar y obtener recursos apropiados a sus necesidades de información; siendo compatible con los registros bajo las AACR2 presentes en los catálogos y adaptables a las bases de datos_emergentes ... Ser un estándar de descripción de recursos que optimice el uso de herramientas en línea, escritas en inglés con la disponibilidad de ser usadas en otros idiomas y de uso fácil y eficiente, como herramienta tanto de ayuda como de entrenamiento]. (2007 p. 2-3)

En otras palabras, puede resultar un estándar universal gracias a sus principios básicos, como los principios de representación usados en la transcripción de los datos, los principios de conveniencia de uso para generar descripciones y notas comprensibles para nuestros usuarios haciendo a los registros bibliográficos, y a los OPAC mismos, herramientas capaces de ofrecer una cobertura completa sobre todos los tipos de contenidos (tomando en cuenta los estándares existentes propios de otras comunidades como son los archivos, museos, editores, la web semántica, etc.,) que además permite la producción de metadatos estructurados para crear búsquedas a través del uso de otras herramientas más avanzadas e interactivas que concedan el acceso buscado por los usuarios y, por ende, la obtención de un amplio rango de recursos de información.

Entonces las RDA (basadas en el modelo conceptual FRBR y observando los principios de catalogación para construir criterios bibliográficos y mejores sistemas para el futuro, que incluyen crear atributos como descriptores para recursos de naturalezas distintas) conjuntamente con los metadatos estructurados se traducen en el vehículo para definir los contenidos de descripción bibliográfica, así como aquellos datos necesarios en una herramienta tecnológica capaz de soportar y traducir la organización de los datos en forma flexible al lenguaje de máquina y los códigos de programación de diversos sistemas ad hoc.

\subsection{Los formatos de codificación}

Con el surgimiento de las RDA se ha cuestionado sí el formato MARC21 bibliográfico (como lenguaje de codificación) es el más apropiado para almacenar la información descrita bajo la lógica de las RDA; la respuesta a este cuestionamiento ha puesto de manifiesto esta carencia. Si bien es cierto que tanto MARC21 como sus antecesores (USMARC, UKMARC, CANMARC, IBERMARC, etc.) cumplen con la norma ISO 2709 en el intercambio de información entre sistemas, esto no ha evitado el surgimiento de nuevas formas de intercambio de información, tales como el XML. Es por ello que, ha surgido una nueva propuesta de codificación llamada Bibliographic Framework Initiative BIBFRAME, en reemplazo de los anteriores (Library of Congress, 2016).

En este sentido, BIBFRAME se presume como la base futura en dónde se reunirán las descripciones bibliográficas cuyo ambiente natural de diseño es la web y que además observa las bondades propias del formato MARC21 junto con las necesidades específicas de los bibliotecólogos. Este modelo ofrece establecer con claridad la diferencia entre los contenidos conceptuales y sus manifestaciones tanto físicas como digitales así como identificar inequívocamente la información de sus entidades, al tiempo que aprovechará y expondrá sus relaciones observando las FRBR, de cara a las RDA y su estructura. 
Lo anterior, por obviedad, debe permitir la citación de datos de la biblioteca como fuente de origen, de la misma forma que sucede ahora con autor y título de cada recurso de información. Se ha mencionado que con BIBFRAME será posible el hospedaje de diferentes modelos de contenido y reglas de catalogación para lo que se establecieron cuatro niveles de mayor rango (que se han venido desarrollando en RDA) de donde:

- BIBFRAME work: identifica la esencia conceptual,

- BIBFRAME instance: refleja la personificación material de una obra,

- BIBFRAME authority: identifica la cosa o concepto asociado a los dos anteriores y

- BIBFRAME annotation: provee una nueva forma de expandir la descripción en apoyo de sus tres antecesores.

Además, se prevé la existencia de un vocabulario específico reusable, basado en la lógica de las ontologías y los requerimientos específicos de los mecanismos y protocolos de intercambio; estos como factores claves en la descripción de recursos ya que su sustento se encuentra en las relaciones que se establecen como norma entre sus elementos. Es decir, obra-obra, obrainstancia, obra-autoridad. En síntesis, BIBFRAME tiende a mejorar el almacenamiento de la información bibliográfica en las bases de datos descriptivas y su extracción para el intercambio y la recuperación a través de la web. Este es el sendero que desde el ámbito bibliotecológico desembocará en un gran aporte para la construcción de contenidos visibles y estructurados en la web semántica.

\section{Las bases de datos descriptivas y su diseño estructural}

Para el desarrollo y creación de las bases de datos existen diferentes modelos, sin embargo, en la actualidad la mayoría de las bases de datos bibliográficas están diseñadas y construidas, principalmente, en el lenguaje de consulta estructurada SQL, mismo que se encuentra estrechamente vinculado con la gestión de las bases de datos relacionales, lo que genera que los manejadores de bases de datos preferidos sean Oracle, PostgreSQL, MySQL y SQL Server. Estas bases de datos, en lenguaje SQL, se han utilizado desde principios de los años 80 , lo que ha permitido registrar una gran cantidad de información descriptiva.

Los programas propios de los Sistemas integrales de automatización para bibliotecas (SIAB) ${ }^{10}$ cuyo inicio se dio con el surgimiento de los Integrated set for information system (ISIS) y el MINISIS vigente en máquinas con ambiente Windows 8 o 10, para después seguir con el desarrollo del Ohio College Library Center (OCLC) ahora conocido como Online Computer Library Center desde 1975 (Goldstein, 1983); ambos, apoyados en el formato MARC en sus versiones I y II respectivamente y su continuo desarrollo en estas dos estructuras lo que facilitó tanto la consulta como el intercambio de información pues cumplían con la norma ISO 2709, ya sea mediante archivos o en línea a través del protocolo de comunicación Z39.50.

10 Autores como Jacquesson (1995) y David (2001) han estudiado el tema de los SIAB, su conformación y la construcción de bases de datos multifuncionales, adecuadaos para la gestión de bibliotecas y la integración de bases de datos de forma que la información se almacena para un uso compartido y específico por cada uno de los módulos que le componen. 
Estas bases de datos descriptivas estaban ligadas con una interface de consulta propia del sistema integral, instalada en las computadoras donde se realizaba la búsqueda y recuperación; esto limitaba la consulta de la base de datos bibliográfica a las computadoras asignadas a esta única tarea dentro de las bibliotecas. Con el surgimiento de internet, y posteriormente de la web, fue evidente la necesidad de crear una interface adecuada a esta nueva forma de publicación, lo que dotó de mayor presencia a los catálogos de las bibliotecas en la web. Este cambio hizo que las interfaces creadas inicialmente para la búsqueda y recuperación de información desaparecieran, llevando a los sistemas integrales de información a crear interfaces web para el catálogo al público.

Otros cambios determinantes en la conformación de las bases de datos descriptivas corresponden al surgimiento de nuevos materiales visuales de imagen fija (con diversos formatos existentes cuyas extensiones pueden ser: .bmp .jpeg .ppt .png .tiff .gif), sonoros (algunos con extensiones: .mp3 .mp4 .wma .wav .aiff .raw .ram .wap), audiovisuales y multimedia (entre otros se encuentran las extensiones: .mp4 .mpeg .wmv .avi .3gp), además que los materiales textuales incrementaron sus formatos de registro físicos (tales como: casetes, discos, disquetes, cintas magnéticas) a electrónicos (todos ellos publicados en la web permitiendo que la información bibliográfica descriptiva se enriqueciera con vínculos o ligas a todas estas nuevas formas de presentar información enunciadas, ya fuera que estuvieran almacenadas en el mismo sistema integral de información o publicadas en servidores diferentes.

En resumen, a lo largo de treinta años se ha visto evolucionar a los SIAB que han pasado de Catálogos de bibliotecas a Portales de información con una mayor presencia y visibilidad dentro de la web, proporcionando servicios a un creciente número de usuarios tanto presenciales como remotos (gracias a que desde los años 80 surgieron nuevas formas de almacenamiento y de publicar la información). Esto permitió que los SIAB pasen de la modalidad "clientes/programas de consulta" a "catálogos web" y esto llevó a establecer más servicios, sin que se registraran cambios en los modelos de creación y desarrollo de las bases de datos descriptivas correspondientes.

Cada cambio que se ha presentado genera una cadena de nuevas necesidades y retos por alcanzar. Los cambios antes descritos se centran en los despliegues (como formas en que se muestran los datos bibliográficos o descriptivos en el ámbito digital), no así en la arquitectura de la información como la parte más estructural acorde a lo que se ha venido desarrollando en la transformación de la web y la manera de proyectarse. Es por esto que las miradas de los bibliotecólogos deberían centrarse en el uso de la lógica respecto a la forma en que se codifica, se logra identificar, publicar y se utiliza la información, ello como una forma de potencializarla y a sus posibles vinculaciones mediante la aplicabilidad y el uso tanto de los metadatos como de las ontologías, las cuáles funcionan como vehículos para visualizar la información para y por los usuarios de la biblioteca digital o híbrida, lo que generará una mayor visibilidad y fuerte presencia dentro de la web.

Todo esto nos lleva a tener claro el cambio de la descripción bibliográfica y de su codificación; en ambos sentidos se han generado las propuestas necesarias que permitirán hacer más eficiente su manejo. Tenemos por un lado la propuesta RDA que permite una mejor descripción 
bibliográfica, y por el otro lado la propuesta BIBFRAME que mejorará la forma de codificar y por lo tanto su publicación en web. Pero hasta aquí se ha hablado poco sobre el diseño y estructura de la base de datos descriptivos que almacenarán estas nuevas descripciones y códigos. Aun cuando actualmente se prevén estos cambios, la estructura de las bases de datos sigue siendo escrita bajo el lenguaje SQL y diseñadas bajo la lógica del Modelo entidadrelación, hecho que para algunos ya resulta ineficaz.

De tal suerte que los bibliotecólogos no podemos soslayar los cambios que se suscitan y tampoco aquellos que se requieren dentro del ámbito de la Ingeniería de Sistemas, sus estructuras y diseños en cuanto a las bases de datos utilizadas por los SIAB. Por eso, ante este escenario de cambio, se plantea la opción de aplicar el Modelo orientado a objetos (MOO) como el más adecuado para diseñar y estructurar las bases de datos de los SIAB, ya que permite una mejor definición de sus elementos dando mayor importancia al objeto y sus relaciones, mismas que nos recuerdan las relaciones semánticas que se establecen mediante la generación, establecimiento y empleo de las ontologías.

\section{Bases para la modelación: El Modelo orientado a objetos en las bases de datos descriptivas}

La base en el Modelo orientado a objetos ${ }^{11}$ se sustenta en la definición de los objetos y el establecimiento de las clases de objetos. Los objetos son entidades físicas o sucesos que se describen en términos de sus datos, procesos y también presentan un estado y un comportamiento. Es decir, se trata de una ocurrencia específica sin que sea el objeto físico mismo, sino la representación conceptual del objeto que consiste básicamente en datos y procesos. Es pues una programación basada en componentes y/o en agentes, en dónde se incorporan nuevas características a las bases de datos descriptivas y a los mecanismos de almacenamiento de objetos de longitudes indeterminadas para que sean capaces de encapsular métodos en su interior, usando estos objetos como datos complejos o como funciones.

Ahora bien, los objetos del mismo tipo se agrupan para formar clases, las cuales consisten en objetos que tienen los mismos atributos y procesos. Una clase de objeto puede describir un elemento del entorno de la institución, un recurso de la institución o una transacción importante; ejemplos de los anteriores son:

- Elementos de entorno: usuario, cliente, proveedor.

- Recursos de la institución: bibliotecólogo, empleado, obra, dispositivo.

- Transacciones importantes: solicitud de compra, solicitud de préstamo, cobro de multa.

11 Sus creadores fueron Kristen Nygaard y Ole-Johan Dahl en la década de los años 60 tratando de describir y simular fenómenos como las redes neuronales, madurando los conceptos básicos de encapsulamiento, atributos de objetos y el reconocimiento de las propiedades comunes de herencia y sentando los principios de expresión de elementos del mundo real, hoy conocidos como entidades clasificadas (cosas tangibles, roles, incidentes, interacciones y especificaciones). Lo cual ha influenciado en el desarrollo de lenguajes modernos en la Programación orientada a objetos. 
Cuando se tienen clases de objetos en las que se determina la interrelación o combinación de los datos con los procesos propios de un objeto se presenta el denominado encapsulamiento que se puede comprender a través de una imagen mental como una caja cerrada con una etiqueta en el exterior en la cual se identifica su contenido. Con este ejemplo se pone de manifiesto que dentro de cada clase encapsulada se cuenta con una "parte de información privada" (datos y procesos específicos que no necesariamente se muestran de forma abierta, pero que se cuenta con el acceso a sus propios datos y al código de programa para realizar su proceso en cada ocurrencia de objeto) y una "parte de información pública" (que se muestra y usa en forma corriente, pues es tanto la definición de aquellos datos que se encuentran disponibles en esa clase de objetos como de aquellos procesos que pueden efectuarse). De modo que estas asignaciones de datos y procesos son lo que dan pie a la existencia del ocultamiento de la información, incluyendo así (por naturaleza propia del MOO) cierto grado de seguridad y control que no existe en los diseños orientados a procesos o a datos, generando un mayor nivel de calidad e integridad en los sistemas. Ejemplo de lo anterior se presenta en el diagrama de la Figura 1.

\section{FIGURA 1}

\section{Ejemplo de construcción de clases de objetos y su diagramación}

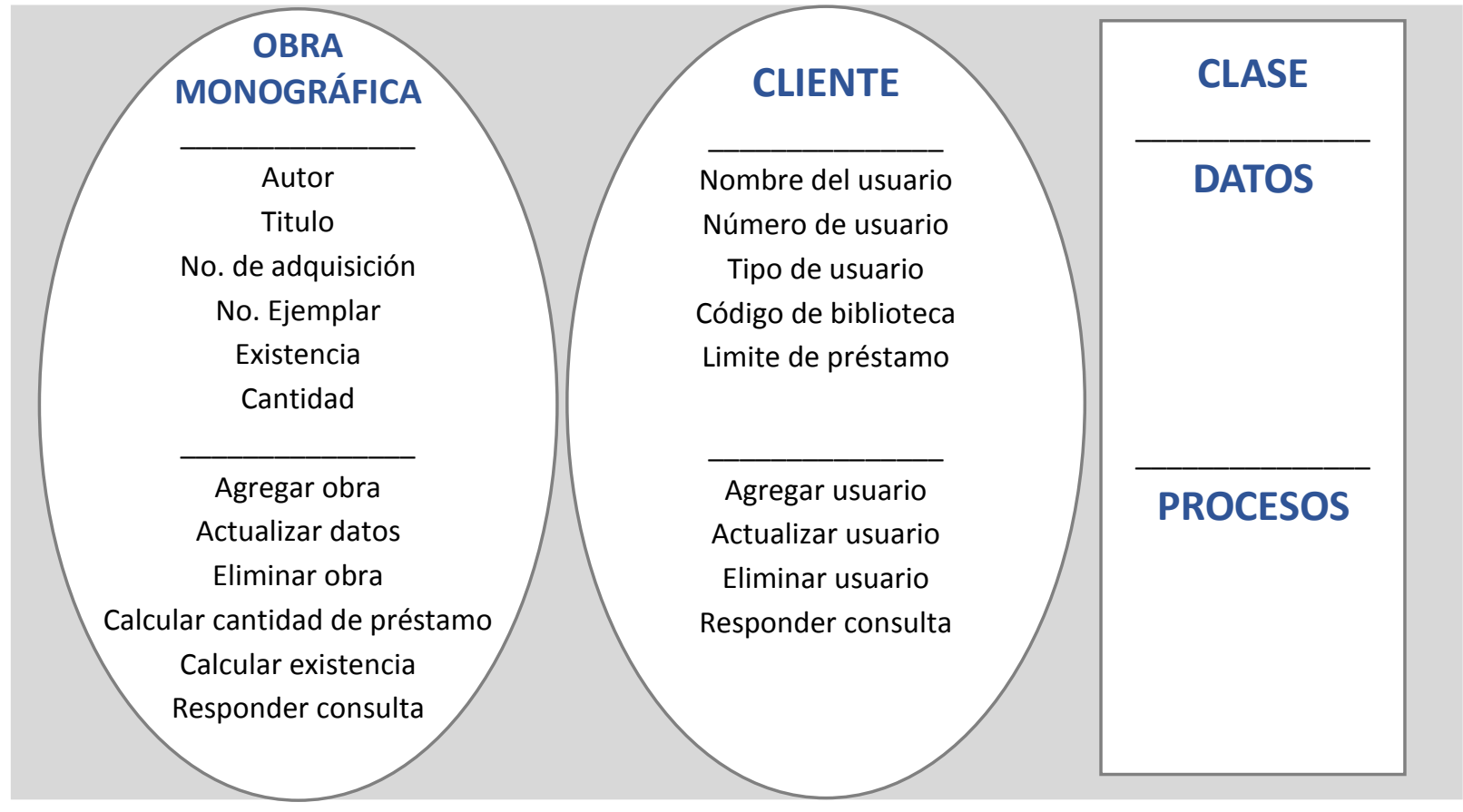

Fuente: Elaboración propia de la autora

Un programa orientado a objetos normalmente se compone de un gran número de estos, y es mediante la interacción de objetos que los programadores pueden definir la funcionalidad de un programa y dar forma a comportamientos más complejos de los que un único elemento puede 
lograr ${ }^{12}$; es por ello que los objetos de software interactúan entre sí enviándose mensajes y especificando las funciones a realizar. ${ }^{13}$

Según Booch, (2001),

La programación orientada a objetos (POO) es un método de implementación, en el cual los programas están organizados como colecciones cooperativas de objetos, cada uno de los cuales representa una instancia de alguna clase y cuyas clases son todas miembros de una jerarquía de clases unidas vía relaciones de herencia" (Booch, 2001, p. 25).

Y de acuerdo con Herrera Delgado (2014) las características primordiales son: "1. usa objetos, no algoritmos, como bloques lógicos de construcción (jerarquía 'parte de') 2. Cada objeto es instancia de alguna clase 3 . Las clases están relacionadas entre sí, vía relaciones de herencia (jerarquía 'tipo de'”). (p. 43)

Con lo cual se evidencia la interrelación de los pasos requeridos para su desarrollo, bajo la lógica orientada a objetos, y la retroalimentación del propio sistema resulta de gran importancia para permitir establecer futuras revisiones y de ser necesario reestructuraciones que permitan alcanzar una mayor eficiencia y/o su mejor adecuación a la realidad de la web semántica.

De esta manera, el cambio vislumbrado en la Bibliotecología involucra a FRBR/RDA y BIBFRAME, mientras que en la Ingeniería de sistemas el cambio de las bases de datos transita del Modelo entidad-relación al Modelo orientado a objetos para su diseño y construcción. Conjugando estos cambios se obtendrá un mejor análisis, diseño y programación de las bases de datos descriptivas que serán utilizadas por los SIAB y todos aquellos sitios de consulta existentes (como los buscadores web), pues al tener su información descrita en RDA y codificada -actualmente- en MARC21 o MARCXML y -en un futuro- en BIBFRAME se podrá extraer bajo una estructura mental (con contenido semántico) más cercana a la usada por los usuarios en la búsqueda y recuperación de los datos.

Por eso es necesario el rediseño de las bases de datos -es decir su análisis, diseño y programación- que permita tener almacenada de forma más eficiente las relaciones semánticas que se utilizarán en la descripción bibliográfica, facilitando al usuario la elección sobre "cual" relación cumple con sus necesidades de información y con esto en mente el primer paso corresponde a la propuesta de un modelo conceptual idóneo. 


\section{Propuesta: Un modelo conceptual orientado a objetos para bases de datos descriptivas en bibliotecas}

Gracias a la conjunción e interrelación de los temas anteriores, propios de la primera etapa, es posible generar la toma de decisiones sobre el esbozo y diseño del modelo que contemple un funcionamiento adecuado. El continuo refinamiento y enriquecimiento en esta segunda etapa puede llevarlo a alcanzar un nivel de detalle, teniendo en cuenta lógicamente los resultados que se prevén obtener.

Como podremos ver más adelante, un modelo conceptual se representa a través de uno o más diagramas en los que se identifican las clases con un alto nivel de abstracción, o de relaciones entre los objetos y/o las instancias. Este o estos diagramas pueden elaborarse en notación Unified Modeling Language $(\mathrm{UML})^{14}$ y hacer uso fundamental de los elementos básicos gramaticales, ortográficos y semánticos de este lenguaje para este tipo de diagrama; esto es clases, objetos, instancias y sus relaciones derivadas (Casas Roma y Conesa I Caralt, 2013).

Para establecer las relaciones y la estructura de la base de datos orientada a objetos usaremos los datos creados en RDA, mismos que se almacenarán observando los principios lógicos de organización conforme al modelo FRBR:

... cumpliendo con las características que tienen las estructuras de las bases de datos orientadas hacia los objetos. Es decir, los elementos de los datos descriptivos serán recolectados en registros que paralelamente cumplan con las indicaciones de las relaciones primarias en el modelo FRBR. (Rodríguez, 2012, p. 174).

\subsection{Creación de los objetos y construcción del modelo}

Aquellos datos que se han establecido como clases contemplan algunas de las entidades primarias de las FRBR, es decir obra, expresión y manifestación; mientras que en el cuarto elemento designado como primario en este modelo corresponde a la descripción. De esta manera, para identificar y distribuir los elementos que se exhiben dentro de las siguientes clases, se tomaron tres documentos para realizar un cruce de información, en los cuales se presentan dos propuestas de desarrollo de las RDA, estos son: las tablas desarrolladas por el 6JSC (JSC, 2015), la propuesta de la LC en torno al desarrollo de estándares (Library of Congress, 2008) y el mapeo desarrollado por Joseph A. Kiegel, director de los servicios de catalogación y metadatos de la Biblioteca en la Universidad de Washington (Kiegel, 2016).

De su comparación se establecieron los elementos básicos y más pertinentes del modelo a proponer, para establecer las clases principales y los objetos que cada una de ellas contendría (ya fuera de forma explícita o encapsulada), asociándolas posteriormente con las instancias existentes o -si fuera el caso- desarrollando nuevas instancias acordes a los objetos,

14 Una herramienta estándar, aceptada internacionalmente, propia de programadores y analistas funcionales, para la creación o el desarrollo de programas, su estudio y la escritura posterior del código informático; es el resultado de los primeros pasos de la programación. 
respetando la sintaxis de SGML. Finalmente, el resultado obtenido tras estas actividades corresponde a la creación de quince objetos para este MOO.

Aprovechando que en esta propuesta se trabaja con objetos, y aun cuando se consideraron las directrices establecidas por el modelo FRBR, es claro que estos objetos permiten modificar la lógica de organización ya conocida y usada, de tal suerte que las instancias que se agruparon en función de los capítulos desarrollados en las RDA aquí no son rigurosamente observadas. En este sentido, la distribución de los descriptores reunidos por las RDA requirió de un procedimiento analítico y del establecimiento de criterios de ordenamiento tales como:

a) Tomar los datos que se concentraban en las tablas antes enunciadas

b) Determinar el tratamiento de los datos resultantes

c) Establecer mecanismos de control para la construcción de las clases

d) Determinar cuáles serán los objetos propios de cada una de las clases

e) Identificar aquellos elementos que se encapsularían, aplicando analogías y estableciendo jerarquías

f) Dar un seguimiento exhaustivo de inclusión y revisión de la lógica aplicada a cada elemento y el cotejo de datos mediante la comparación entre el origen y el producto

g) Evaluar la estructura de las clases y los objetos que las componen, así como su eficacia de construcción en función de la lógica propia de las bases orientadas a objetos, representadas en los modelos.

Ahora bien, para establecer una representación homogénea de los quince objetos y sus elementos estructurales (es decir, las clases, objetos e instancias) se establece el esquema presentado en la Figura 2, en forma genérica, aplicando el lenguaje UML.

\section{FIGURA 2}

Ejemplo de diagrama de una clase de objeto en lenguaje UML

\begin{tabular}{|c|c|c|c|c|}
\hline Clase & $\begin{array}{l}\text { El primer apartado hace referencia a la clase, identificada } \\
\text { por un concepto específico, mismo que se consideró como } \\
\text { el más general y representativo para que diera cabida a los } \\
\text { elementos del siguiente apartado. }\end{array}$ \\
\hline Objetos & $\begin{array}{l}\text { El segundo apartado enuncia y reúne a los objetos, ya sea } \\
\text { de forma explícita o de forma implícita, gracias al } \\
\text { encapsulamiento. }\end{array}$ \\
\hline Instancias & $\begin{array}{l}\text { El tercer apartado hace referencia a la instancia de cada } \\
\text { objeto previamente considerado, como un paso que nos } \\
\text { acerca a la fase de programación. }\end{array}$
\end{tabular}

Fuente: Elaboración propia de la autora.

Centrando nuestra atención en los siguientes esquemas, podemos generar una lectura en la que el conjunto de objetos que se presentan en cada uno de estos cuadros conforman, por un 
lado, una clase de objeto pues contienen los nombres de los datos que se representarán dentro de ella (información que permite identificarla) mientras que, por otro lado, gracias a las instancias se establece el nombre que le corresponderá a cada objeto, dentro del lenguaje de marcado (las tags) y el lenguaje de programación del software.

Cada una de las 15 clases de objeto, se identifica con el término designado, en idioma inglés y guarda el orden, tal como se visualiza en la Tabla 1.

TABLA 1

Listado de clases de objetos que conforman la propuesta del Modelo conceptual orientado a objetos

\begin{tabular}{|l|l|l|}
\multicolumn{3}{|c|}{ Clases propuestas para el Modelo Orientado a Objetos de una Base de } \\
Datos Descriptiva \\
\hline 1. Work & 6. Acces & 11. Publisher \\
\hline 2. Description & 7. Manifestation & 12. Editor/on \\
\hline 3. Copyrigth & 8. Manifacturer & 13. Distributor \\
\hline 4. Control number & 9. Title & 14. Performer \\
\hline 5. Expression & 10. Creator & 15. Subject \\
\hline
\end{tabular}

Fuente: Elaboración propia de la autora.

Ahora bien, para mostrar la forma en que se generan los encapsulamientos dentro de los objetos que se han creado para este modelo, podemos tomar como ejemplo el esquema de la clase Manifestation que a razón de su amplitud se describe en forma horizontal, sin perder el orden en que se anuncian sus apartados y que además con la Figura 3 se explicitan los elementos que contiene. 
FIGURA 3

Ejemplo de diagrama de representación de la clase de objeto Manifestation propuesta en el Modelo conceptual orientado a objetos y sus elementos encapsulados

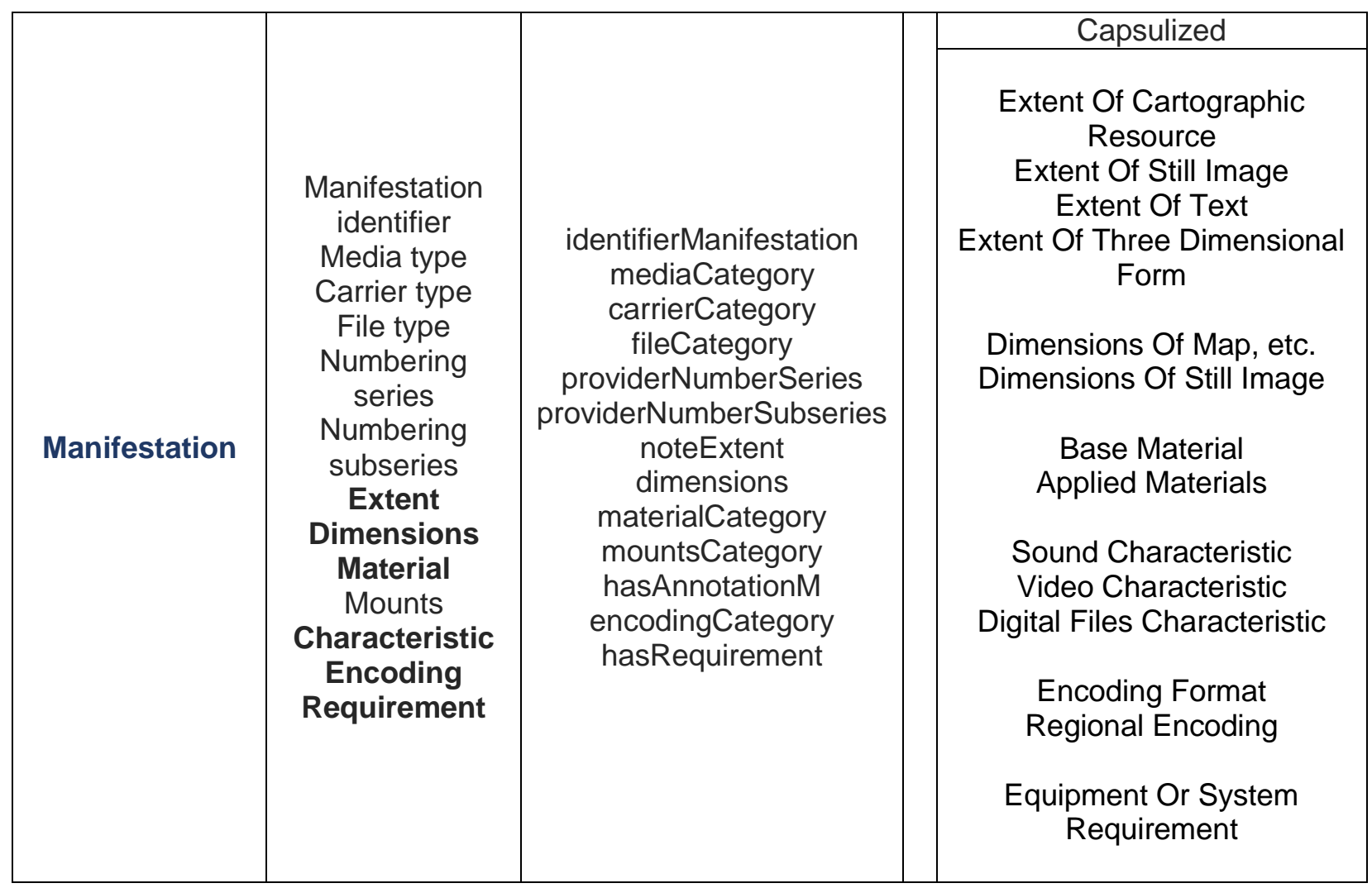

Fuente: Elaboración propia de la autora.

Como se puede apreciar, en la columna izquierda se identifica el nombre de la clase en cuestión, la siguiente columna exhibe los objetos que corresponden a la clase y de entre ellos se puede apreciar aquellos que se encuentran en negritas, estos objetos contienen los elementos que se describen en la columna de la derecha a los cuales encapsula, tenemos entonces lo siguiente:

- Dentro de Extent se concentran:

- Extent of cartographic resource

- Extent of still image

- Extent of text

- Extent of three dimensional form

- Dentro de Dimensions se concentran:

- Dimensions of map, etc.

- Dimensions of still image 
- Dentro de Material se concentran:

- Base material

- Applied materials

- Dentro de Characteristic se concentran:

- Sound characteristic

- Video characteristic

- Digital files characteristic

- Dentro de Encoding se concentran:

- Encoding format

- Regional encoding

- Dentro de Requirement se concentran:

- Equipment or system requirement

Es claro que aquello que se encapsula está estrechamente vinculado con el objeto determinado y que refiere a un nivel de especificidad mayor, por lo que es susceptible de simplemente añadirse en caso de ser necesario.

Y finalmente, se aprecia la columna en la cual se establecieron las instancias a través de las cuales los objetos se vuelven lenguaje máquina.

\subsubsection{El Modelo de obra}

Todas las clases congregadas bajo el esquema o modelo de obra ponen de manifiesto que es en WORK de dónde se desprende la existencia de las catorce clases restantes, de tal suerte que éstas se encuentran supeditadas a su existencia, de acuerdo con el esquema generado que se presenta como la Figura 4. 
FIGURA 4

Esquema en UML del modelo WORK, como propuesta del Modelo conceptual orientado a objetos

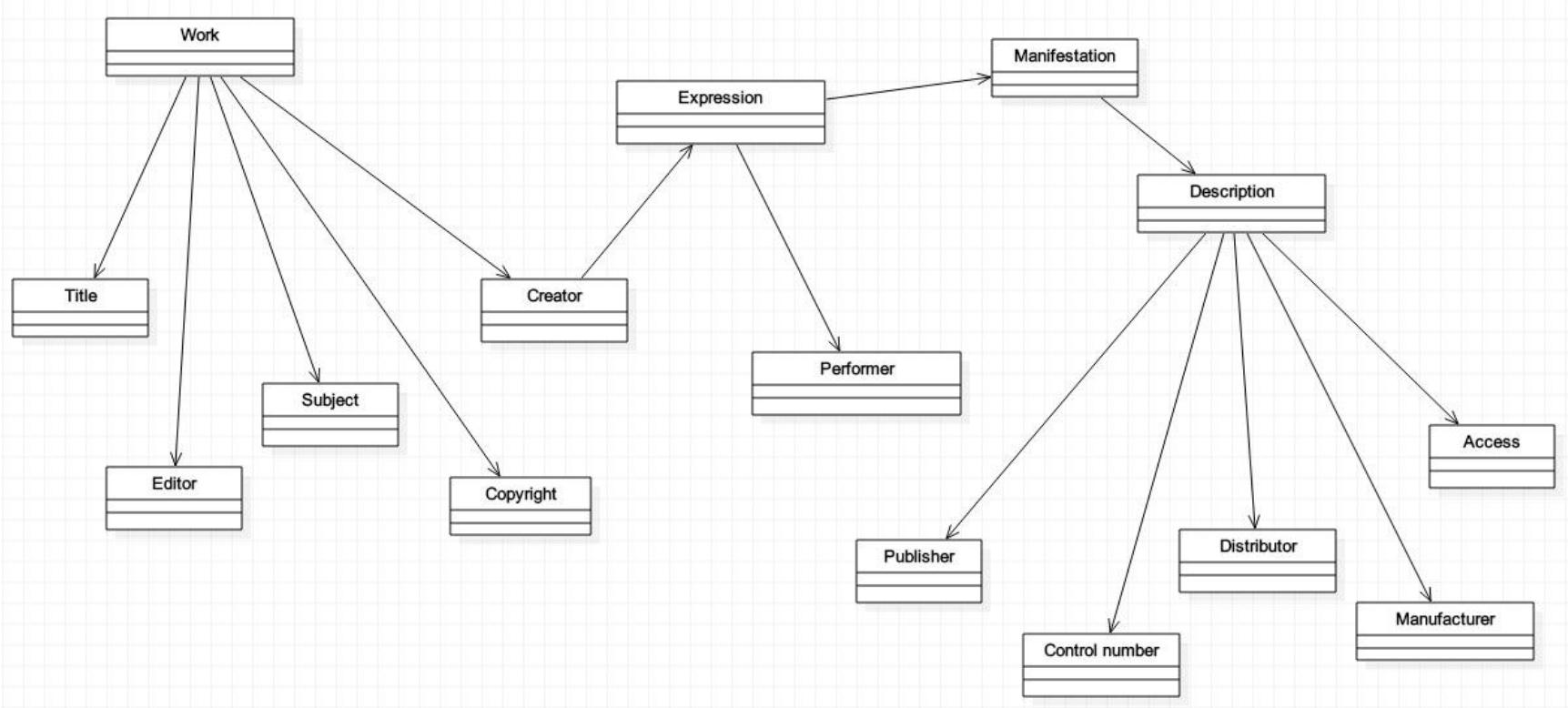

Fuente: Elaboración propia de la autora.

En un primer acercamiento, este modelo permite esclarecer la organización jerárquica dentro de las clases de objeto creadas para determinar su primacía o dependencia, poniendo de manifiesto sus relaciones, a través de las cuales se desarrolla el flujo y el procesamiento de la información. Esta es la principal razón por la cual, en apariencia, resulta un modelo distinto de los que le secundan.

\subsubsection{El Modelo de dominio}

Ahora bien, el esquema o Modelo dominio está planteado en términos completamente conceptuales; permite presentar una forma distinta de organización y agrupamiento de las clases y evidencia a los objetos que las componen como elementos básicos para la descripción de los recursos de información, observando la realidad digital y el contexto actual de las bibliotecas en la web.

Como se puede apreciar en la Figura 5, se enlistan los objetos propios de cada clase, así como las relaciones que deben establecerse entre ellos. Claramente se aprecia que el punto de partida, nuevamente, es la Obra y como clases más altas le acompañan Expresión, Manifestación y Descripción, cuya vinculación se establece en forma horizontal, seguida de la especificación de los objetos. Para el resto de las clases se evidencia la dependencia directa y la relación/comunicación que debe tener con los objetos restantes. 
FIGURA 5

Esquema en UML del modelo DOMINIO, como propuesta del Modelo conceptual orientado a objetos

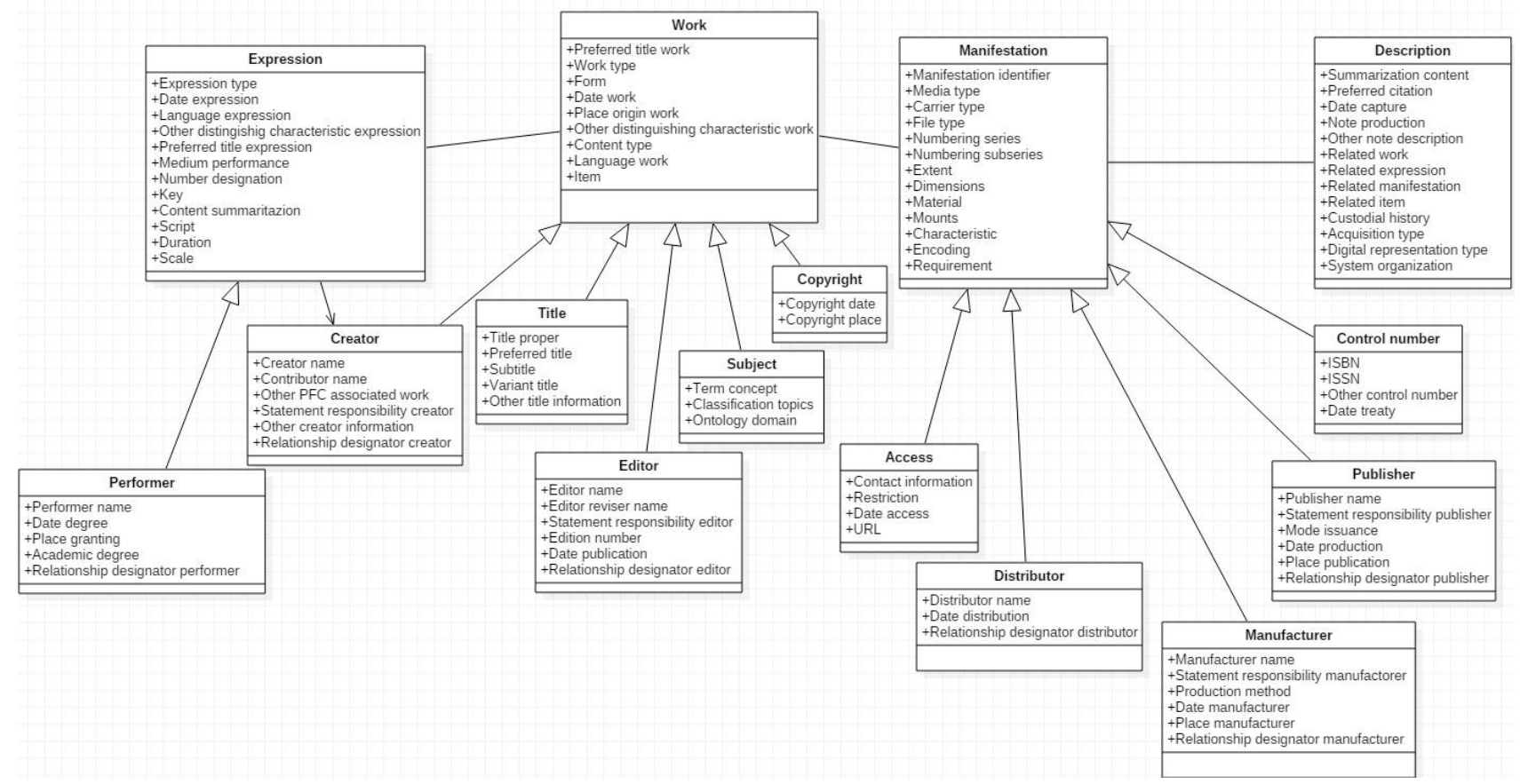

Fuente: Elaboración propia de la autora.

A este modelo se le puede calificar como el corazón del Diseño conceptual orientado a objetos que se propone, ya que permitirá tener presentes a todos los elementos a ser considerados dentro del esquema programático de nuestra base de datos en forma sintetizada, organizada y clara.

\subsubsection{El Modelo de instancia}

Finalmente, el interés por desarrollar el esquema o Modelo instancia responde a la elaboración y presentación de las tags o instancias para cada objeto y, con ello, hacer evidente la viabilidad esbozada mediante el esquema o modelo dominio. Este esquema resulta una forma de comprobar la viabilidad, aplicabilidad y validez de la propuesta teórica de este proyecto de investigación interdisciplinario. También muestra en lenguaje de programación una forma de identificar cada objeto con sus relaciones. Además, la Figura 6 permite generar las conclusiones que a continuación se enuncian. 
FIGURA 6

Esquema en UML del modelo INSTANCIA, como propuesta del Modelo conceptual orientado a objetos

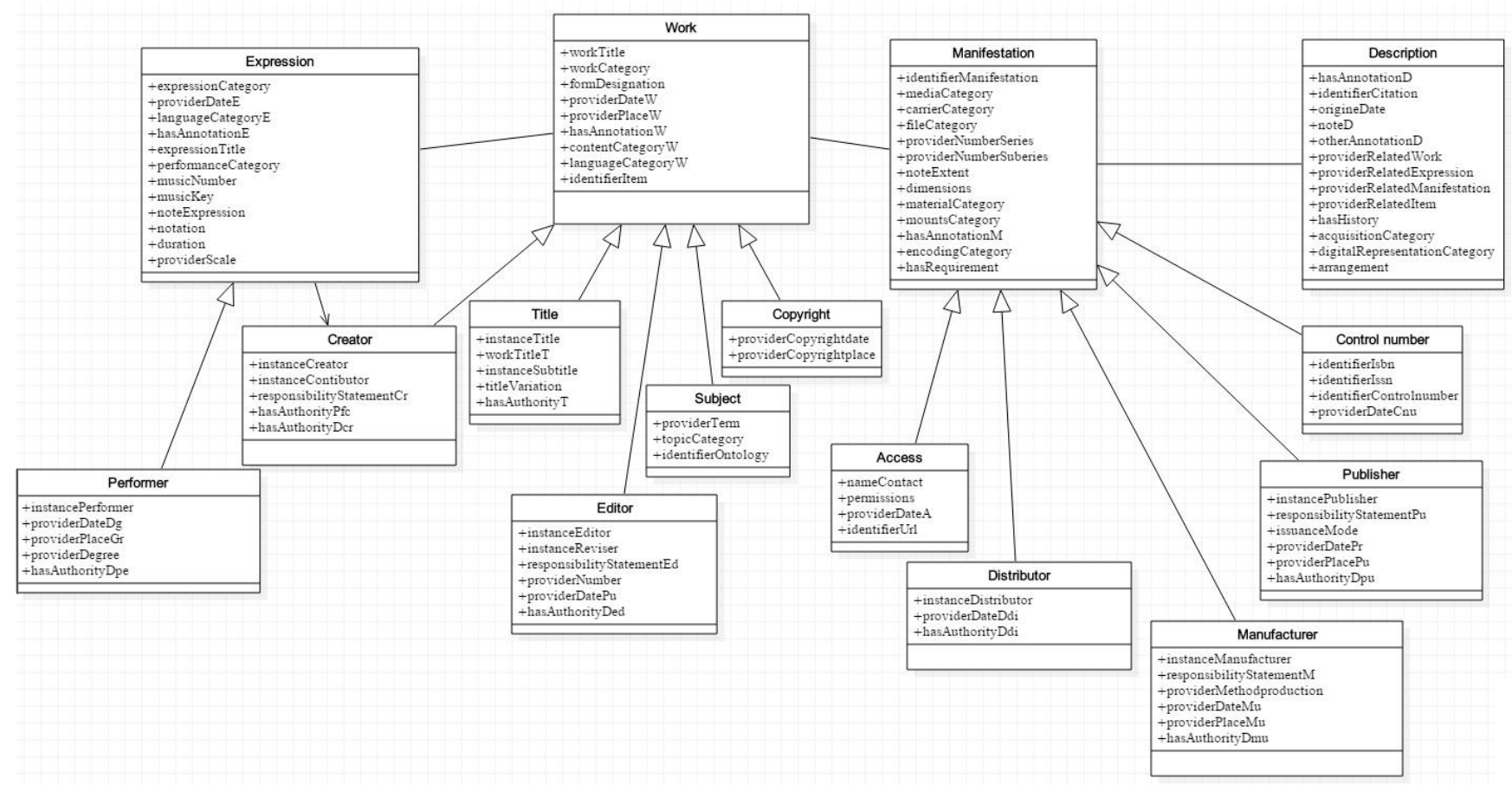

Fuente: Elaboración propia de la autora.

Ya que se han incluido todos los elementos a considerar, se desarrollan las instancias que nos permitirán iniciar el proceso de programación en las bases esenciales para su reconocimiento particular (único) y ser insertadas, las instancias, en los lenguajes de programación para generar la base de datos correspondiente.

Con la creación y conjunción de estos tres modelos (obra, dominio e instancia), se logra esbozar un horizonte diferente a los existentes en relación a la estructuración y conformación de las bases de datos descriptivas para las bibliotecas digitales, bajo una nueva lógica en dónde se incluyen los nuevos fundamentos y códigos de descripción de recursos de información y los principios de programación de la web.

\section{Conclusiones}

Los cambios que en la última década se han suscitado en las normas para describir y codificar la información generan cuestionamientos respecto de las actuales bases de datos descriptivas que almacenan información bibliográfica. La biblioteca académica, ha centrado su atención en los metadatos para la generación de ontologías, aplicando la semanticidad ${ }^{15}$ y colaborando con 
los objetivos propuestos por las FRBR, lo que origina cambios en los datos bibliográficos que se han de llevar hacía el ambiente de la web semántica y su descubrimiento más allá de los catálogos.

Ante la necesidad de contar con herramientas tecnológicas que permitan no solo manejar un volumen inmenso de información sino también capaces de recuperarla de acuerdo a las necesidades específicas de cada usuario, es que se plantea la necesidad de modificar la forma de diseñar y estructurar las bases de datos descriptivas; específicamente aquellas utilizadas por los SIAB.

Esta es una de las pautas para que, en el ámbito de la Ingeniería de Sistemas, se vuelquen las miradas nuevamente sobre el Modelo orientado a objetos como la opción más adecuada para el diseño de las bases de datos requeridas en la actualidad por las bibliotecas digitales. En dichas bases, las RDA resultan el elemento imprescindible a través del cual se simplifica el diseño y modelado estructural de las bases de datos, dotándoles de orden, criterios, datos y metadatos que mejor describan los recursos de información presentes en la web y en su construcción semántica en forma sencilla. En este grupo de códigos de organización y bases de datos orientadas a objetos se conjugan las normas y estándares internacionales preexistentes como son: MARC, ISBD, AACR2, las funciones básicas de los OPAC, DCMI, Uniform Resource Identifier (URI), además del Open Metadata Register (OMR) para el desarrollo de metadatos, creados por términos ontológicos y vocabularios controlados todos ellos montados en la web de Berners-Lee presentada en 2009 (TED, 2009).

La generación de modelos permite representar la teoría y las condiciones ideales en las que se produce un fenómeno, permitiendo verificar una ley o una teoría, al tiempo que constituye una muestra particular en la explicación teórica general muy cercana a la realidad.

A través de este Modelo de base de datos orientada a objetos se busca representar el comportamiento de los objetos en las condiciones ideales de una generación de base de datos descriptiva, como una muestra particular de todas las explicaciones dadas en términos teóricos y generales simplificando la propuesta, mostrando aspectos importantes, evidenciando la referencia que se traduce a la teoría en realidad y facilitando su comprensión. El que se haya definido que el presente modelo debía relacionar lo abstracto con lo concreto se debe a que el modelo básico se constituye con base en los conceptos y sus relaciones. Adicionalmente, este modelo califica como formal gracias al nivel en el que se presenta la estructura idealizada como análoga al sistema real, lo que permite su predicción.

Cabe hacer notar que este desarrollo tenderá hacía la web semántica ya que sus principales componentes incluidos en los objetos corresponden a las ontologías, lo que de entrada plantea el cambio de los motores de búsqueda en el mundo digital y las lógicas aplicadas en las formas de búsqueda que el usuario final emplea en la propia web.

Esta es la razón fundamental que propicia la generación de la propuesta de un modelo a partir de la realidad global en que nos vemos inmersos los bibliotecólogos. El bibliotecólogo es el agente encargado de desarrollar la gestión del dato, de la información, del conocimiento, así como de su diseminación en el ámbito digital (mediante los desarrollos y las propuestas para organizar la información desde su esfera). Es por ello que resulta una voz muy valiosa durante 
la creación y desarrollo de propuestas que deberán impactar en el modelado de programas con una estructura orientada a objetos, en la que se creen bases de datos bibliográficasdescriptivas cada vez más flexibles.

De los tres modelos que se esbozan en el presente artículo, el tercero denominado como Modelo de instancia se percibe fuera de la etapa de diseño, por lo que se ofrece como un producto práctico que puede resultar el inicio de una tercera etapa en la que tendría lugar la implementación del modelo propuesto mediante la programación dirigida a la generación del software; gracias a que las instancias propuestas en este modelo vuelven semánticos los datos, haciéndolos legibles tanto para el hombre como para la máquina gracias a su especificación.

De acuerdo con los datos que proporciona el Instituto Nacional de Estadística y Geografía (INEGI, 2016) a mediados del 2015 en México, se registraron 62.4 millones de personas que usan los servicios ofrecidos por internet. Mientras que, en el mundo, la UNESCO estima que más de 3.5 billones de personas tienen acceso a internet lo que puede representar una producción diaria de por lo menos 2.5 trillones de bytes de datos en la web para finales del 2016 (UNESCO, 2016). Por si fuera poco, en lo que resta de esta década, se calcula que los dispositivos tecnológicos se quintuplicarán en número.

Con esto se puede aseverar que la tecnología no espera a nadie y si este ritmo de desarrollo continuo prevalece, las bibliotecas se encuentran más que obligadas en aprender a manejar el poder disruptivo de la tecnología desde dentro, lo que se convierte en el mejor escenario prospectivo posible para hacerle frente al reto de la transformación digital. Alcanzar su máxima capitalización mediante el desarrollo y aplicación del Modelo conceptual orientado a objetos para la Biblioteca digital aquí presentado, y teniendo como objetivo la óptima operación de las reglas de negocio para bibliotecas y su flujo propio de información, generarían de manera consecuente la modificación de algunos de sus productos (específicamente los catálogos, los motores de búsqueda y el despliegue de una información más vinculada) y de sus servicios ofrecidos a la comunidad primaria estrechando su relación con los bibliotecólogos, lo que llevará a una mayor visualización y presencia en la web.

\section{Referencias}

Alegsa, L. (2016) Diccionario de informática, internet, tecnologías y computación on line. Santa Fe, Argentina: ALEGSA. Recuperado de http://www.alegsa.com.ar/Dic/agente_de_software.php

Ashby, W. R. (1957). An introduction to Cybernetics. Londres: Chapman \& Hall. Recuperado de http://pespmc1.vub.ac.be/books//ntroCyb.pdf

Batini, C., Ceri, S., y Navathe, S. B. (1992). Diseño conceptual de bases de datos: un enfoque de entidades-interrelaciones. Estados Unidos: Addison-Wesley y Díaz de Santos. 
Berners-Lee, T., Hedler, J., y Lassila, O. (mayo, 2001) The Semantic Web. Scientific American, 284(5), 34-45 Recuperado de https://wwwsop.inria.fr/acacia/cours/essi2006/Scientific\%20American_\%20Feature\%20Article_\%20 The\%20Semantic\%20Web_\%20May\%202001.pdf

Bertalanffy, L. V. (1976). Teoría de los sistemas: fundamentos, desarrollo y aplicaciones. México: FCE.

Booch, G. (2001) Análisis y diseño Orientado a Objetos con aplicaciones. Estados Unidos: Addison-Wesley y Diaz de Santos.

Casas Roma, J., y Conesa I Caralt, J. (2013). Diseño conceptual de bases de datos en UML. Barcelona: Editorial UOC.

David, L. T. (2001). Introduction to Integrated Library Systems. Module 2 of the ICT for Library and Information Professionals. Bangkok, Tailandia: UNESCO, Information and Informatics Unit. Recuperado de http://arizona.aws.openrepository.com/arizona/bitstream/10150/106374/1/125105e.pdf

Goldstein, Ch. M. (julio, 1983). Integrated Library Systems. Bulletin of the Medical Library Association, 7(3), 308-311. Recuperado de http://www.ncbi.nlm.nih.gov/pmc/articles/PMC227197/pdf/mlab00067-0079.pdf

Herrera Delgado, L. B. (abril, 2014). Modelación, web semántica y biblioteca. En A. A. Leiva Mederos \& Y. Hidalgo Delgado (Presidencia). Taller de Web semántica. Simposio llevado acabo en el Congreso International de Información INFO 2014, La Habana, Cuba. Recuperado de http://ceur-ws.org/Vol-1219/paper4.pdf

Instituto Nacional de Estadística y Geografía. (13 de mayo, 2016). Estadística a propósito del día mundial de Internet (17 de mayo). Aguascalientes, México: Autor. Recuperado de http://www.inegi.org.mx/saladeprensa/aproposito/2016/internet2016_0.pdf

Jacquesson, A. (1995). L'informatisation des bibliothèques: historique, stratègie et perspectives. Paris, Francia: Cercle de la Librairie.

Játiva Miralles, M. V. (2009). El catálogo: un recurso en expansión. Anales de documentación, (12), 69-91. Recuperado de http://www.redalyc.org/pdf/635/63511932004.pdf

Joint Steering Committee for Development of RDA. (1 de noviembre, 2007). Strategic plan for RDA 2005-2009. Recuperado de http://www.rdajsc.org/archivedsite/docs/5strategic1 rev2.pdf

Joint Steering Committee for Development of RDA. (setiembre/octubre, 2015). Complete Examples- Bibliographic Records. Recuperado de http://www.rdatoolkit.org/sites/default/files/6jsc_rda_complete_examples_bibliographic_r evisedoct2015.pdf 
Kiegel, J. (29 de agosto, 2016). Mapping of RDA Core to BIBFRAME 2.0. Estados Unidos: University of Washington. Recuperado de http://faculty.washington.edu/kiegel/

Kunder, Maurice de. (9 de febrero, 2016). WorldWideWeb.com: Daily Estimated Size of the World Wide Web. Noord-Brant, Netherlands: Tilburg University. Recuperado de http://www.worldwidewebsize.com/

Library of Congress, Development and MARC Standards Office. (24 de abril, 2008). MARC to Dublin Core Crosswalk. Estados Unidos: Autor. Recuperado de http://www.loc.gov/marc/marc2dc.html

Library of Congress. (21 de abril, 2016). Overview of the BIBFRAME 2.0 Model. Estados Unidos: Autor. Recuperado de https://www.loc.gov/bibframe/docs/bibframe2-model.html

Prebor, G. (01 de diciembre, 2010). Analysis of the Interdisciplinary Nature of Information and Library Science. Journal of Librarianship and Information Science, 42(4), 256-267.

Rodríguez García, A. A. (enero/abril, 2012). Claves para la implementación de los lineamientos recursos, descripción y acceso. Investigación Bibliotecológica, 26(56), 159-178. Recuperado de http://www.scielo.org.mx/pdf/ib/v26n56/v26n56a8.pdf

Sánchez López, S. E. (2007). Modelo de indexación de formas en sistemas VIR, basado en ontologías (tesis de maestría inédita, Universidad de las Américas Puebla). Recuperado de http://catarina.udlap.mx/u_dl_a/tales/documentos/mcc/sanchez_I_se/capitulo4.pdf

Taylor, A. G. (2000). Understanding FRBR: What it is and how it will affect our retrieval tools. Connecticut, Estados Unidos: Libraries Unlimited.

TED. (31 de mayo, 2009). El futuro de la Web 1/2 [archivo de video]. Recuperado de https://www.youtube.com/watch?v=MEjRFXbqjLc

Tillet, B. (2004). ¿Qué es FRBR? Un modelo conceptual del universo bibliográfico. Washington, Estados Unidos: The Library of Congress.

UNESCO. (setiembre, 2016). The State of Broadband 2016: Broadband Catalyzing Sustainable Development. Recuperado de http://unesdoc.unesco.org/images/0024/002459/245905e.pdf

\section{Agradecimientos}

La autora reconoce y agradece el apoyo recibido por CONACYT a través de la beca nacional para cursar estudios de posgrado. 
Herrera Delgado | Volumen 7, número 2, Artículo teórico 1, Jul-Dic, 2017 | e-Ciencias de la Información

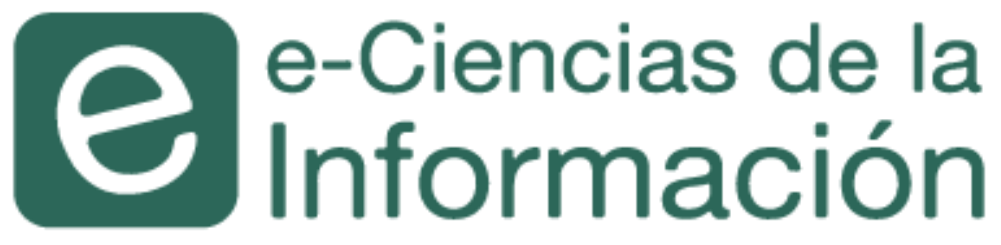

¿Desea publicar su trabajo? Ingrese aquí

O escríbanos a la siguiente dirección: revista.ebci@ucr.ac.cr

(ㄷ)(1)(2)

\section{1}

Origen: respuesta a una necesidad

En el año 2011, la Escuela de

Bibliotecología y Ciencias de la Información (EBCI) de la Universidad de Costa Rica (UCR) reconoció la importancia de crear nuevas y mejores alternativas para difusión de la investigación. e-Ciencias de la Información es la respuesta a un contexto actual marcado por una mayor apertura, flexibilidad, y rigurosidad en la verificación de los datos y su procesamiento.

\section{3}

Revista de la UCR

e-Ciencias de la Información es una revista científica que aborda las nuevas temáticas de desarrollo e investigación en las Ciencias de la Información, en el ámbito nacional e internacional. Así, colabora significativamente en el progreso de esta disciplina. Por sus parámetros de calidad, pertenece al grupo de las revistas más importantes de la UCR y se encuentra ampliamente indizada en los importantes catálogos.

\section{En la actualidad}

\section{Posicionamiento internacional}

Se encuentra en el Cuartil A del UCR Índex para el 2017, posicionándola como una de las mejores revistas de la Universidad de

Costa Rica, un reflejo claro y conciso sobre

su calidad y trascendencia en el área

apoyado por otros hitos como su ingreso a

Scielo, DOAJ, Latindex y otros.
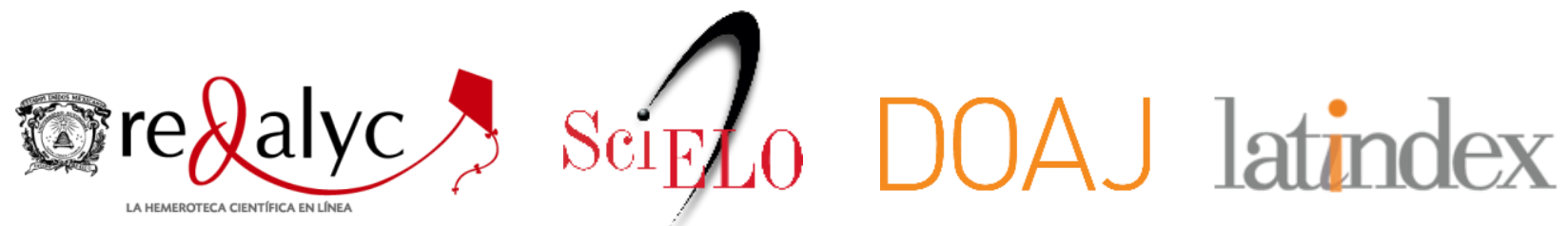

e-Ciencias de la Información está indexada en los catálogos más prestigiosos.

Para conocer la lista completa de índices, ingrese aquí 